\title{
Synchronous malignancies in patients with breast cancer
}

\author{
Beata Sas-Korczyńska ${ }^{1}$, Jerzy W. Mituś ${ }^{2}$, Wojciech Kamzol ${ }^{1}$, Marta Kołodziej Rzepa², \\ Jerzy Jakubowicz ${ }^{3}$, Wojciech M. Wysocki²
}

\begin{abstract}
Introduction. The continuously improving cancer detection at an early stage and improving survival rates have been observed and, therefore, patients are predisposed to detection of multiple primaries. It has been reported that the incidence of multiple primaries in breast cancer patients ranges from of $4 \%$ to $17 \%$.
\end{abstract}

Materials and methods. A group of 112 breast cancer patients with synchronous malignancies was presented. They constituted $0.09 \%$ of patients ( 118,952 cases) who were treated for breast cancer at the same time period, and made up $3.5 \%$ of all patients (3,176 cases) with multiple primary cancers, and $21.7 \%$ of all patients (517 cases) with breast cancer who developed multiple primaries.

Results. The most frequent type of synchronous primary malignancy was breast cancer (63.4\%) and $90.1 \%$ of them were diagnosed at the same time or within one month following the first breast cancer diagnosis. Among cases of non-breast synchronous primaries, female genital organ malignancies were predominant (36.6\%). Synchronous breast cancer was diagnosed significantly earlier than non-breast cancers (mean time was 0.4 and 1 month, respectively, $p=0.0123$ ). Better results in the group with synchronous contralateral breast cancer in comparison to synchronous breast and non-breast cancer were observed (5-year overall survival rates were $90.9 \%$ and $66.3 \%$, respectively, and 5 -year disease-free survival rate $-62.5 \%$ and $51.3 \%$, respectively).

NOWOTWORY J Oncol 2017; 67, 6: 336-341

Key words: synchronous malignancies, breast cancer, contralateral breast cancer

\section{Introduction}

Significant progress has been observed in the prevention, diagnosis and therapy of cancers. Screening programmes and improvements in diagnostic methods result in cancer being detected at an earlier stage and, moreover, the advances in treatment methods yield an increase the overall survival of cancer patients. In consequence, the occurrence of multiple independent primary cancers in the same patient is still growing and ranges from $1 \%$ to $18 \%$ [1-7].

The definition of multiple primary cancers is based on the classic criteria of Warren and Gates which include: (i) the site of origin, (ii) the morphology and histology of tu- mours, and (iii) the amount of time passed since the initial diagnosis. This means that tumours arise in other organs than the independent primaries, each tumour has to be histologically distinctive and the possibility of metastasis or recurrence must be excluded [8,9]. Considering the time of occurrence, multiple primary cancers generally fall into the synchronous or metachronous [8-10]. According to the International Agency for Research on Cancer (IARC), the diagnosis of synchronous primaries is made within an interval of less than 6 months [8].

Multiple primary cancers are categorized according to predominant etiologic influences: treatment-related, syn-

${ }^{1}$ Clinic of Oncology, Maria Skłodowska-Curie Memorial Cancer Centre and Institute of Oncology, Kraków Branch, Poland ${ }^{2}$ Department of Surgical Oncology, Maria Skłodowska-Curie Memorial Cancer Centre and Institute of Oncology, Kraków Branch, Poland

${ }^{3}$ Department of Gynecological Oncology, Maria Skłodowska-Curie Memorial Cancer Centre and Institute of Oncology, Kraków Branch, Poland 
dromic and those due to shared etiologic factors [11]. Causal mechanisms of their development include the following: (i) host factors - genetic (BRCA mutations, Li-Fraumeni syndrome), hormonal, prior cancer diagnosis and treatment exposures, (ii) lifestyle factors such as alcohol and tobacco use (risk factors for several cancer types), and (iii) environmental influences - geography (areas of increased radon exposure), pathogens (human papilloma virus or Epstein-Barr virus infections) and occupational factors [1, 3, 8, 10, 12-14].

Breast cancer is the most common cancer in women; in Poland 17,400 new cases of breast cancer (which constituted $21.7 \%$ of all cancers in 2014) were diagnosed in [15]. In these patients, continuously improving detection at an early stage and improving survival rates have been observed and, therefore, these patients are predisposed to detection of multiple primaries. It has been reported that the incidence of multiple primaries in breast cancer patients ranges from of $4 \%$ to $17 \%[5,7,10,16]$.

The risk of developing multiple primary malignancies in breast cancer patients is associated with positive family history, suppressor gene mutation (BRCA1, BRCA2, PTEN, TP53), age, body weight, alcohol intake, hormonal replacement therapy and breast cancer therapy (especially for metachronous primaries) [14, 17, 18].

Lee et al. showed that synchronous malignancies developed in $23.8 \%$ of breast cancer patients and the most frequent were malignancies located in the thyroid gland (71\% cases) and in the gynaecologic tract (9.7\%) [8].

The purpose of this study was (i) to present the characteristics of breast cancer patients with synchronous primaries in relation to the type of synchronous primaries (breast and breast versus breast and non-breast) and (ii) to evaluate the outcomes and the impact of the type of synchronous cancers on survival rate.

\section{Materials and methods}

In this paper, synchronous malignancies are defined as primaries diagnosed at the same time or following the diagnosis of breast cancer in a time interval shorter than 6 months.

\section{Patient population}

Between 1965 and 2014, synchronous malignancies (in compliance with the above definition) were diagnosed in 112 breast cancer patients who were treated at Maria Skłodowska-Curie Memorial Cancer Centre and Institute of Oncology in Kraków. These patients constituted $0.09 \%$ of all patients $(118,952$ cases $)$ who were treated for breast cancer in this time period, and they made up 3.5\% of all patients $(3,176$ cases) with multiple primary cancers and $21.7 \%$ of all patients (517 cases) with breast cancer who developed multiple primaries.

Table I presents the types of synchronous malignancies and Figure 1 presents the time interval between the diagnosis of breast cancer and synchronous malignancy in the analysed group of 112 breast cancer patients.

The mean time interval between diagnosis of breast carcinoma and synchronous cancers was $0.6 \pm 1.1$ month (range: $0-5$ months). More than $80 \%$ of synchronous malignancies were diagnosed at the same time or during the first month following breast cancer diagnosis.

The most frequent type of synchronous primary malignancy was breast cancer (71 cases - 63.4\%). In 64 cases, this type of second synchronous cancer was diagnosed at

Table I. The types of synchronous primary cancers in 112 patients with breast cancer

\begin{tabular}{|c|c|c|c|}
\hline Diagnosis of synchronous cancers & ICD-10 code & No. of cases & $\%$ \\
\hline Total & & 112 & 100.0 \\
\hline Breast cancer (in contralateral breast) & $\mathrm{C} 50$ & 71 & 63.4 \\
\hline Female genital organ cancers: & & 15 & 13.4 \\
\hline Cervical uteri cancer & C53 & 10 & 8.9 \\
\hline Endometrial cancer & C54 & 1 & 0.9 \\
\hline Ovarian cancer & C56 & 4 & 3.6 \\
\hline Digestive organ cancers: & & 2 & 1.8 \\
\hline Stomach cancer & C16 & 1 & 0.9 \\
\hline Rectal cancer & $\mathrm{C} 20$ & 1 & 0.9 \\
\hline Skin cancer & $\mathrm{C} 44$ & 11 & 9.8 \\
\hline Lung cancer & C34 & 1 & 0.9 \\
\hline Urinary tract cancers: & & 4 & 3.6 \\
\hline Kidney cancer & C64 & 3 & 2.7 \\
\hline Urinary bladder cancer & $\mathrm{C} 67$ & 1 & \\
\hline Thyroid cancer & $\mathrm{C} 73$ & 3 & 0.9 \\
\hline Lymphatic tissue neoplasms & $\mathrm{C} 81, \mathrm{C} 85, \mathrm{C} 91$ & 4 & 3.6 \\
\hline Laryngeal cancer & C32 & 1 & 0.9 \\
\hline
\end{tabular}




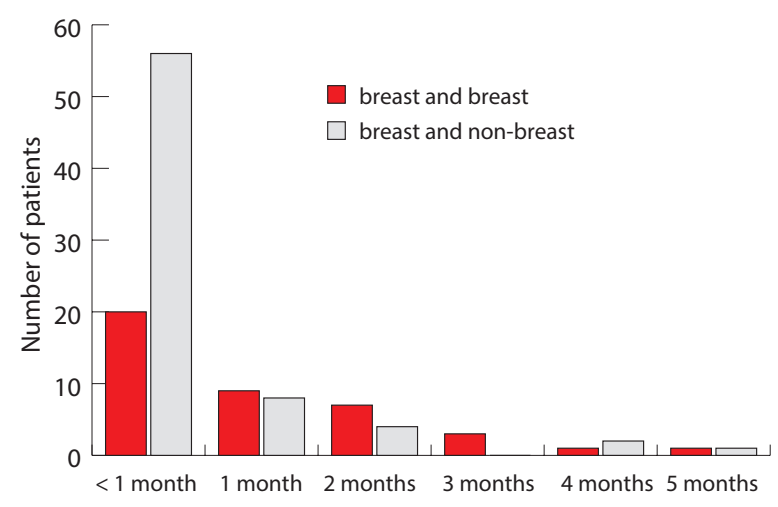

Figure 1. The time interval between the diagnosis of breast cancer and synchronous malignancy in 112 patients

the same time or within one month following first breast cancer diagnosis. Among 41 cases of non-breast synchronous cancers, female genital organ malignancies were predominant ( 15 cases - 36.6\%).

\section{Statistical methods}

In the studied population, two groups were distinguished: patients with synchronous bilateral breast cancer and patients with synchronous non-breast cancer. The comparison of these groups was performed in order to assess the relation to frequency of the analysed factors with the use of the Pearson's Chi-square test for independence (for categorized variables) and variance analysis with Student's t-test (for continuous variables). Overall survival rate and disease-free survival rate were evaluated with the Kaplan-Meier method, and the log-rank test was applied to assess the influence of type of synchronous cancers on the evaluated results.

All calculations were performed by STATISTICA v.12 software (StatSoft, Inc. Tulsa, OK, USA) and the significance level at $\alpha=0.05$ was adopted for all statistical analyses.

\section{Results}

\section{Patient characteristics and comparison}

The mean age at diagnosis of breast cancer was $61.5 \pm$ 12.8 years (range: $30-85$, median: 62 years). Age over 50 years was noted in 90 cases (80.4\%). Positive cancer history was found in 47 patients (42\%) including 16 cases (34\%) with breast cancer history. Synchronous breast cancer appeared in 33 patients with positive family history (70\%) and in 11 patients with breast cancer family history (68.8\%). Benign breast diseases and/or hormonal disturbances were found in 8 individuals (7.2\%).

Because patients with breast cancer and synchronous cancers were managed over a period of 49 years, the diagnostic methods and the spectra of treatment changed along with the indications. Nevertheless, surgery was the primary treatment modality of breast cancer. Because in the presented group of 112 patients with breast cancer and synchronous cancers in 71 cases synchronous bilateral breast cancer was diagnosed, therefore, in our material in 112 patients 183 breast primary tumours were found. Radical mastectomy was performed in 131 breast tumours (71.6\%), whereas 52 tumours (28.4\%) underwent breast-conserving surgery. The following adjuvant therapy was applied: postoperative radiotherapy (77 breast tumours $42.1 \%$ in 55 patients - 49.1\%), chemotherapy (51 patients - 45.5\%) and hormonotherapy (87 patients - 77.7\%), which in 85 patients $(75.9 \%)$ was combined with tamoxifen. In some cases, multimodal treatment was performed in an adjuvant setting.

Table II presents the characteristics of 112 patients with breast cancer and synchronous primary malignancies and their comparison in relation to the type of synchronous primary cancers: breast and breast cancers versus breast and non-breast cancers.

Synchronous breast cancer was diagnosed significantly earlier than non-breast cancers; mean time was 0.4 and 1 month, respectively $(p=0.0123)$ after first breast cancer.

There were no significant differences between the two analysed patients' groups (synchronous primaries: breast and breast cancer versus breast and non-breast cancer) in relation to epidemiological, clinical and pathological features.

\section{Survival and outcomes}

The follow-up period after the diagnosis of breast cancer ranged from 2 to 371 months with a mean value of $78.2 \pm 72$ months and a median of 56.5 months. Table III presents the outcomes which developed during clinical observation. The mean time of these outcomes was as follows: $14.9 \pm 13.9$ months (for locoregional recurrence), $46.6 \pm 44.2$ months (for distant metastases) and $41.1 \pm 33.5$ months (for metachronous carcinomas) after diagnosis of breast cancer. Patients with synchronous contralateral breast cancer had a tendency to more frequent development of distant metastases (32.4\% patients vs $13.8 \%$ patients with synchronous breast and non-breast cancer, $\mathrm{p}=0.07776, \mathrm{Chi}^{2}$ test). However, there were no significant differences $\left(\mathrm{p}>0.05, \mathrm{Chi}^{2}\right.$ test) between the two analysed groups in relation to the development of locoregional recurrences or metachronous (third and fourth) primaries.

During the follow-up period 28 patients (25\%) died, and the causes of death were as follows: breast cancer (13 cases), non-breast primaries (6 cases), coexistent non-cancer internal disease (3 cases), and unknown (6 cases).

The estimated 5-year and 10-year survival rates were $81 \%$ and $65.3 \%$ (for overall survival) and $58.3 \%$ and $44.9 \%$ (for disease-free survival).

Figure 2 shows the probability of overall survival (a) and disease-free survival (b) in relation to the type of synchronous primaries (synchronous contralateral breast cancer versus breast and non-breast cancer). 
Table II. The characteristics of 112 patients with breast cancer and synchronous malignancies and its comparison in relation to the type of synchronous primary cancers: breast and breast cancers versus breast and non-breast cancers

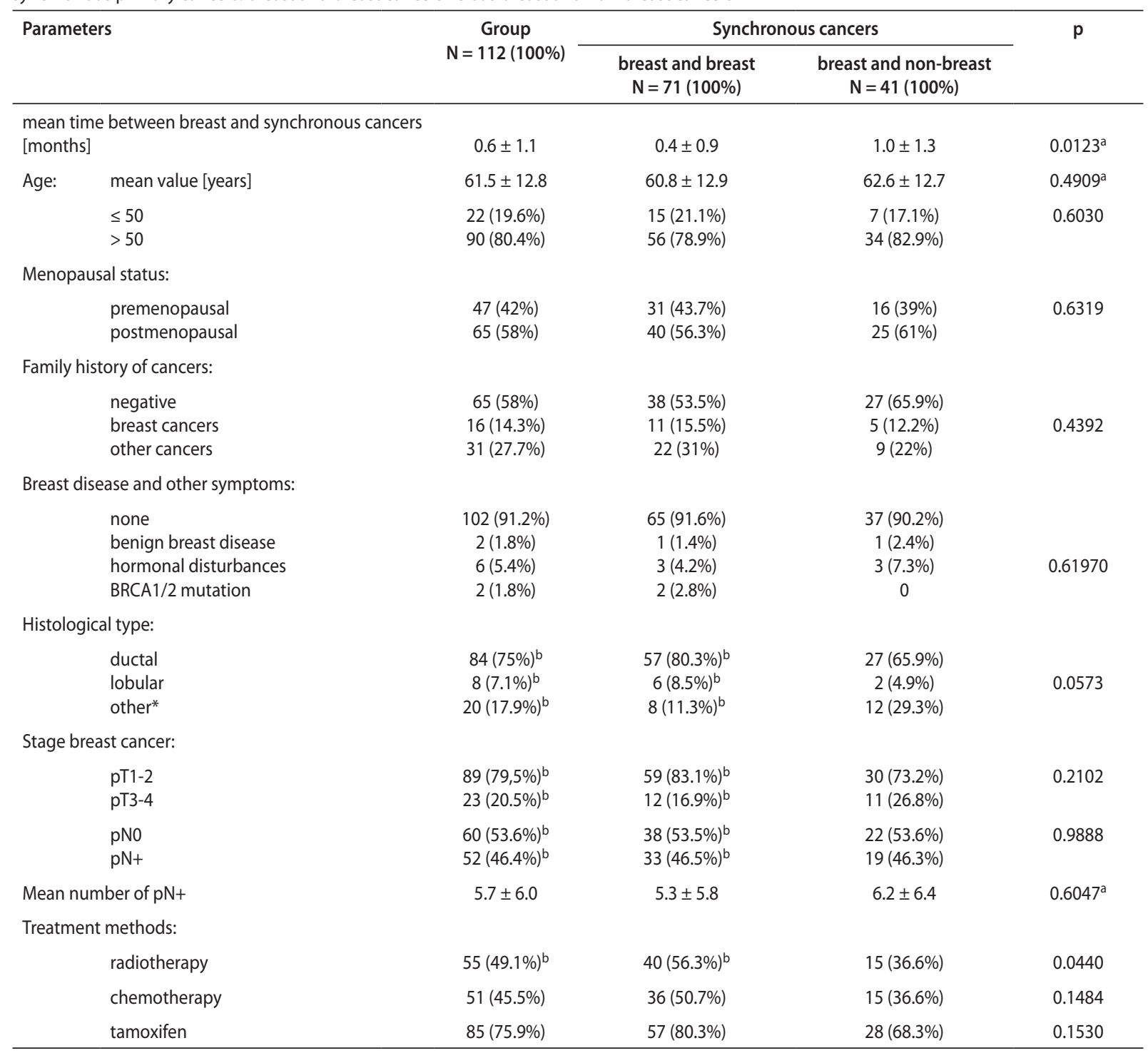

*other types of breast cancer: medullary, mucinous, metaplastic, apocrine, tubular, inflammatory, Paget's disease of the nipple, papillary; ${ }^{\mathrm{a}} \mathrm{p}$ value from $\mathrm{t}$-Student test, other $\mathrm{p}$ for chi-square test; ${ }^{b}$ presence of the parameter in one breast cancer at least (patients with synchronous bilateral breast cancer)

Better results in the group with synchronous contralateral breast cancer in comparison to synchronous breast and non-breast cancer were observed (5-year overall survival rates were $90.9 \%$ and $66.3 \%$, respectively, and 5 -year disease-free survival rate $-62.5 \%$ and $51.3 \%$, respectively), but these observations were statistically nonsignificant.

\section{Discussion}

Published data indicate that $4-17 \%$ of breast cancer patients develop multiple primary malignancies and, according to Lee et al., synchronous primaries were diagnosed in 24\% of cases [5, 7, 8, 10, 16]. The group of 112 patients with breast cancer and synchronous malignancies presented in this study constituted $21.7 \%$ of all patients with breast cancer who developed other primaries in the analysed period. It is interesting that 11 (9.8\%) of our breast cancer patients with synchronous malignancies developed metachronous malignancies during the follow-up period, in comparison to $2.8 \%$ observed in patients analysed by Lee at al. [8].

Our observation showed that the most frequent synchronous neoplasms were contralateral breast cancer (71 cases - 63.4\%) and genital organ cancer ( 15 cases - 13.5\%), of which cervical uterine cancer was predominant (8.9\%).

Some literature data showed that a lobular component of breast carcinoma was associated with an almost 2-fold increase in the risk of developing contralateral breast cancer, especially synchronous bilateral breast cancer [19-21]. 

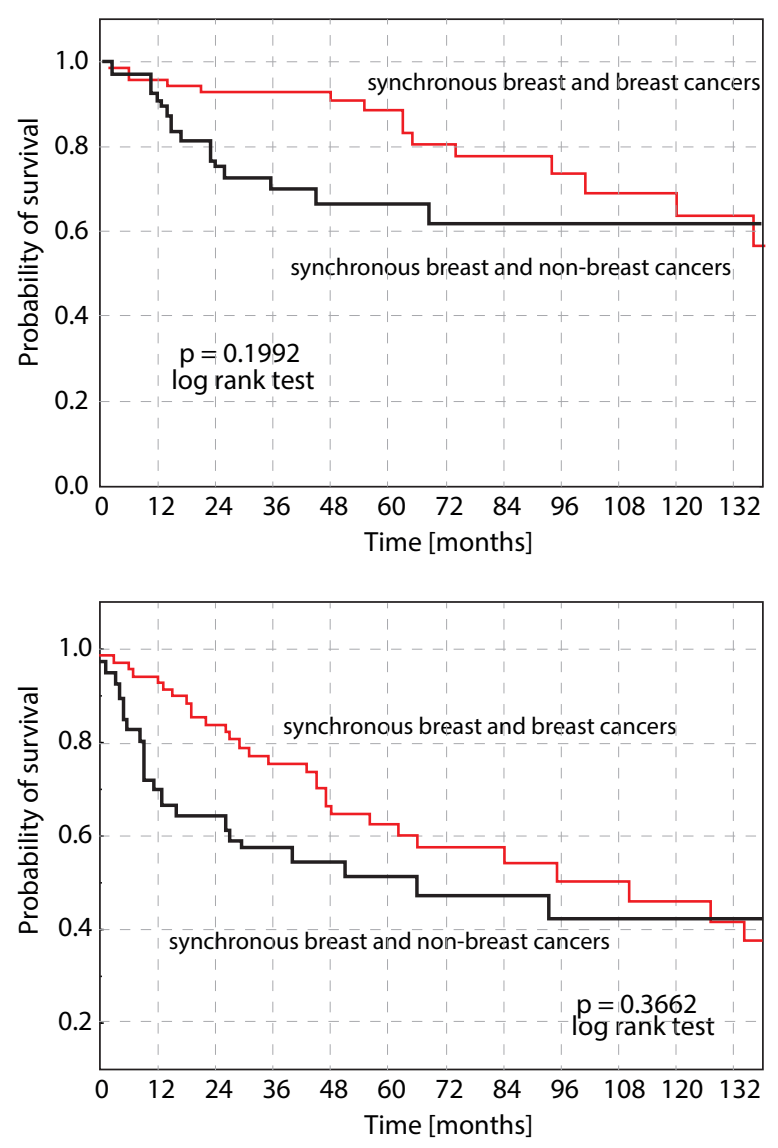

Figure 2. The probability of overall (a) and disease-free (b) survival rates in relation to type of synchronous malignancy in 112 patients treated for breast cancer

Our observations confirmed the tendency of developing synchronous contralateral breast cancer in lobular breast carcinoma patients; in $75 \%$ of cases of lobular breast carcinoma, synchronous contralateral breast cancer developed. Claus et al. observed that patients with lobular breast carcinoma were 2.6 times more likely to be diagnosed with synchronous contralateral breast cancer than patients with ductal breast carcinoma [22].

Published data indicate that age as well as menopausal status at breast cancer diagnosis are risk factors for development of second cancer [18, 19, 21, 23-27]. Lv et al. showed that $84.6 \%$ patients with synchronous malignancies were over 50 years old [27]. In our patients, $80 \%$ of patients ( 90 of 112 cases) were over 50 years old and most of them (65 cases - 58\%) were in postmenopausal status. The comparison to breast cancer patients who developed metachronous malignancies confirmed that patients with synchronous malignancies were significantly older and in postmenopausal status ( $p=0.00001$ and $p=0.00275$, respectively).

The occurrence of a second primary is associated with a significantly increased risk of death and significantly worse survival $[8,28,29]$. Particularly, this regards synchronous
Table III. The outcomes in 112 patients with breast cancer and synchronous malignancies

\begin{tabular}{lcc}
\hline Outcomes & No. of patients & $\%$ \\
\hline Recurrence at chest wall & 10 & 8.9 \\
Distant metastases & 30 & 26.8 \\
bones & 13 & 11.6 \\
liver & 7 & 6.3 \\
lungs & 5 & 4.5 \\
lymph nodes & 4 & 3.6 \\
brain & 3 & 2.7 \\
skin & 1 & 0.9 \\
Metachronous cancers & 11 & 9.8 \\
breast cancer & 1 & 0.9 \\
endometrial cancer & 2 & 1.8 \\
ovarian cancer & 1 & 0.9 \\
skin cancer & 2 & 1.8 \\
kidney cancer & 1 & 0.9 \\
bladder cancer* & $1^{*}$ & $0.9^{*}$ \\
Hodgkin's lymphoma & 1 & 0.9 \\
ocular melanoma & 1 & 0.9 \\
biliary tract cancer* & $1^{*}$ & $0.9^{*}$ \\
\hline
\end{tabular}

*as $4^{\text {th }}$ cancers, and remain - as $3^{\text {rd }}$ morbidity

cancers [27-30]. Results published by Carmichael et al. showed that significantly worse overall survival is observed among patients with synchronous contralateral breast cancer in comparison to metachronous or unilateral breast cancer [31]. Quan et al published that the 5-year survival rate was $80.3 \%$ for synchronous breast cancer and $90.5 \%$ for metachronous breast cancer [32]. Our results were similar and 5 -year survival reached $81 \%$ in all breast cancer patients with synchronous malignancies and $90.9 \%$ in the synchronous contralateral breast cancer subgroup. The five-year survival rate evaluated in breast cancer patients with metachronous malignancies (presented in our earlier publication) was $91.8 \%$ (all breast cancer patients and metachronous malignancies) and $92.9 \%$ in patients with metachronous contralateral breast cancer [33]. However, these results were not statistically significant ( $p=0.27772$, log rank test).

The occurrence of multiple synchronous primaries is challenging and often causes a therapeutic dilemma in daily clinical practice. Therapeutic strategy for patients with synchronous malignancies should be discussed in a multidisciplinary team. The main focus in the therapeutic decision-making process should be placed on (i) evaluation of the prognosis and (ii) the chance of a curative or palliative approach [10].

\section{Conclusions}

1. The most frequent type of synchronous malignancy in patients with breast cancer is contralateral breast cancer (63.3\% of cases) followed by female genital organ cancer (13.4\% of cases).

2. The possibility of development of synchronous primary cancer in breast cancer patients indicates the necessity 
of conducting precise diagnostic procedures, because detection of coexistent cancers influences the choice of optimal treatment strategy.

\section{Conflict of interest: none declared}

\section{Beata Sas-Korczyńska, MD, PhD}

Maria Skłodowska-Curie Memorial Cancer Centre and Institute of Oncology

Kraków Branch

Clinic of Oncology

ul. Garncarska 11

31-115 Kraków, Poland

e-mail:z5korczy@cyf-kr.edu.pl

\section{Received: 9 Nov 2017}

Accepted: 11 Jan 2018

\section{References}

1. Travis LB. The epidemiology of second primary cancers. Cancer Epidemiol Biomarkers Prev 2006; 15: 2020-2026.

2. Trentham-Dietz A, Newcomb PA, Nichols HB et al. Breast cancer risk factors and second primary malignancies among women with breast cancer. Breast Cancer Res Treat 2007; 105: 195-207.

3. Vogt A, Schmid S, Heinimann K et al. Multiple primary tumours: challenges and approaches, a review. ESMO Open 2017; 2: e000172 (doi: 10.1136/esmoopen-2017-000172).

4. Coyte A, Morrison DS, McLoone P. Second primary cancer risk - the impact of applying different definitions of multiple primaries: results from a retrospective population-based cancer registry study. BMC Cancer 2014; 14: 272.

5. Weir HK, Johnson CJ, Thompson TD. The effect of multiple primary rules on population-based cancer survival. Cancer Causes Control 2013; 24: 1231-1242.

6. Rosso $S$, De Angelis R, Ciccolallo L et al. Multiple tumours in survival estimates. Eur J Cancer 2009; 45: 1080-1094.

7. Luciani A, Ascione G, Marussi D et al. Clinical analysis of multiple primary malignancies in eldery. Med Oncol 2009; 26: 27-31.

8. Lee J, Park S, Kim S et al. Characteristics and survival of breast cancer patients with multiple synchronous or metachronous primary cancers. Yansei Medical J 2015; 56: 1213-1220.

9. Filali K, Hedelin G, Schaffer P et al. Multiple primary cancers and estimation of the incidence rates and trends. Eur J Cancer 1996; 32A: 683-690.

10. Amer $\mathrm{MH}$. Multiple neoplasms, single primaries, and patient survival. Cancer Manag Res 2014; 6: 119-134.

11. Travis LB, Rabkin C, Brown LM et al. Cancer survivorship - genetic susceptibility and second primary cancers: research strategies and recommendations. J Nat/ Cancer Inst 2006; 98: 15-25.

12. Mariotto $A B$, Rowland JH, Ries LA et al. Multiple cancer prevalence: a growing challenge in long-term survivorship. Cancer Epidemiol Biomarkers Prev 2007; 16: 566-571.

13. Calip GS, Law EH, Ko NY. Racial and ethnic differences in risk of second primary cancers among breast cancer survivors. Breast Cancer Res Treat 2015; 151:687-696.
14. Molina-Montes E, Requena M, Sanchez-Cantalejo E et al. Risk of second cancers cancer after a first primary breast cancer: a systematic review and meta-analysis. Gynecol Oncol 2015; 136: 158-171.

15. Wojciechowska U, Olasek P, Czuderna K et al. Nowotwory złośliwe w Polsce w 2014; Cancer in Poland in 2014. Warszawa: Krajowy Rejestr Nowotworów, 2016.

16. Kim JY, Song HS. Metachronous double primary cancer after treatment of breast cancer. Cancer Res Treat 2015; 47: 64-71.

17. Evans HS, Lewis CM, Robinson D et al. Incidence of multiple primary cancers in a cohort of women diagnosed with breast cancer in southwest England. Br J Cancer 2001; 84: 435-440.

18. Schaapveld M, Visser O, Louwman MJ et al. Risk of new primary nonbreast cancers after breast cancer treatment: a Dutch population-based study. J Clin Oncol 2008; 26: 1239-1246.

19. Chen $Y$, Thompson W, Semenciw R et al. Epidemiology of contralateral breast cancer. Cancer Epidemiol Biomarkers Prev 1999; 8: 855-861.

20. Horn PL, Thompson WD. Risk of contralateral breast cancer: associattions with histologic, clinical and therapeutic factors. Cancer 1988; 62: 412-424.

21. Hislop TG, Elwood JM, Coldman AJ et al. Second primary cancer of the breast: incidence and risk factors. Br J Cancer 1984; 49: 79-85.

22. Claus EB, Stowe M, Carter D et al. The risk of contralateral breast cancer among women diagnosed with ductal and lobular breast carcinoma in situ: data from the Connecticut Tumor Registry. Breast 2003; 12: 451-456.

23. Liu J, Jiang W, Mao $\mathrm{K}$ et al. Elevated risks of subsequent endometrial cancer development among breast cancer survivors with different hormone receptor status: a SEER analysis. Breast Cancer Res Treat 2015; 150: 439-445.

24. Buist DS, Abraham LA, Barlow WE et al. Diagnosis of second breast cancer events after initial diagnosis of early stage breast cancer. Breast Cancer Res Treat 2010; 124: 863-873.

25. $\mathrm{Li} \mathrm{Cl}$, Malone $\mathrm{KE}$, Porter PL et al. Epidemiologic and molecular risk factors for contralateral breast cancer among young women. Br J Cancer 2003; 89: 513-518.

26. Bernstein JL, Thompson WD, Risch $\mathrm{N}$ et al. Risk factors predicting the incidence of second primary breast cancer among women diagnosed with a first primary breast cancer. Am J Epidemiol 1992; 136: 925-936.

27. Lv M, Zhang X, Shen Y et al. Clinical analysis and prognosis of synchronous and metachronous multiple primary malignant tumors. Medicine (Baltimore) 2017; 96: e6799.

28. Schmid SM, Pfefferkorn C, Myrick ME et al. Prognosis of early-stage synchronous bilateral invasive breast cancer. Eur J Surg Oncol 2011; 37: 623-628.

29. Holm M, Tjonneland A, Balslev E et al. Prognosis of synchronous bilateral breast cancer: a review and meta-analysis of observational studies. Breast Cancer Res Treat 2014; 146: 461-475.

30. Amer MH. Multiple neoplasms, single primaries, and patient survival. Cancer Manag Res 2014; 6: 119-134.

31. Carmichael AR, Bendall S, Lockerbie $L$ et al. The long-term outcome of synchronous bilateral breast cancer is worse than metachronous or unilateral tumors. Eur J Surg Oncol 2002; 28: 388-391.

32. Quan G, Pommier SJ, Pommier RF. Incidence and outcomes of contralateral breast cancers. Am J Surg 2008; 195: 645-650

33. Sas-Korczyńska B, Kamzol W, Kołodziej Rzepa M et al.The characteristics of breast cancer patients with metachronous primary malignancies. Nowotwory J Oncol 2017; 67: 14-23. 